\title{
Internalization of Mycobacterium bovis, Bacillus Calmette Guerin, by bladder cancer cells is cytotoxic
}

\author{
SIM-HWEE POOK, JUWITA NORASMARA BTE RAHMAT, \\ KESAVAN ESUVARANATHAN and RATHA MAHENDRAN \\ Department of Surgery, Yong Loo Lin School of Medicine, National University of Singapore, Republic of Singapore
}

Received May 25, 2007; Accepted July 18, 2007

\begin{abstract}
Instillation of Bacillus Calmette Guerin (BCG) into the bladder is the standard treatment for superficial bladder cancer. It leads to a local inflammatory response due to the release of cytokines and influx of immune cells to the tumor site. Although the presence of an intact immune system is an essential criterion for successful therapy, attachment of the bacteria to the bladder urothelial is just as important. The purpose of our study is to determine the role of bacterial internalization by epithelial cells. Transfection of the $\alpha 5$ integrin gene into the BCG unresponsive bladder cancer cell line, RT4, caused an increase in bacterial uptake and also increased cell death. Treatment of cells with cycloheximide did not prevent bacterial internalization but blocked its cytotoxic effect suggesting that unlike cell death, the process of bacterial internalization does not require new protein synthesis. Our data also show that the bacteria secretory products can prevent its own internalization. The extract prepared from lyophilized BCG altered the phosphorylation status of the focal adhesion kinase which is responsible for cellular endocytosis. Therefore, bacterial phosphatases may be present in the bacterial extract. Their activity may inhibit BCG internalization. Thus washing the reconstituted bacteria to remove the enzymes before instillation into the bladder might improve the therapeutic outcome of intravesical BCG therapy.
\end{abstract}

\section{Introduction}

Superficial bladder cancer is characterized by frequent recurrences and in some cases progression to muscle invasive or metastatic disease. The gold standard for the treatment of bladder cancer is transurethral resection of the bladder tumor followed by intravesical instillations of Bacillus Calmette Guerin (BCG). The use of BCG in bladder cancer is the most

Correspondence to: Dr Ratha Mahendran, Department of Surgery, Yong Loo Lin School of Medicine, National University of Singapore, 5 Lower Kent Ridge Road, Singapore 119074, Republic of Singapore E-mail: surrm@nus.edu.sg

Key words: Bacillus Calmette Guerin, focal adhesion kinase, integrins, bladder cancer cells successful immunotherapy known. However, despite its reported efficacy, a number of patients do not respond to therapy.

The exact mechanism by which BCG mediates its antitumor effect remains unclear. In addition to the requirement for an intact immune system, direct contact between BCG and urothelial cells is mandatory (1). The binding of BCG to the urothelium requires the participation of the $\alpha 5$ and $\beta 1$ integrins (2). $\alpha 5 \beta 1$ integrins are receptors for fibronectin and are involved in the internalization of a number of bacteria in mammalian cells. This attachment is a prerequisite for successful therapy and is supported by a number of studies. When BCG attachment was abolished by pre-treatment with anti-fibronectin antibody, the BCG-induced immune response was prevented (3). Disruption of the binding of BCG to the bladder urothelium by modification of the BCG fibronectin attachment protein (FAP) also interfered with the expression of the BCG-induced anti-tumor response (4). In vitro, a study using different grades of human bladder cancer cell lines with differential ability to internalize BCG showed that cells with a greater ability to internalize BCG were more responsive to the cytotoxic effects of BCG (5). In addition, bacterial uptake is required for BCG-induced secretion of interleukin-6 (IL-6) by bladder cancer cells (6) and increases the expression of cell surface antigens such as major histocompatibility complex (MHC) Class II, CD 1, CD 8 and intercellular adhesion molecule I (ICAM I) (7).

We previously reported that internalization of $\mathrm{BCG}$ correlates with a drop in cellular redox status (8). In this study, we show that by transient transfection of the integrin $\alpha 5$ receptors, we are able to induce cell death in 2 cell lines that are not responsive to $\mathrm{BCG}$ cytotoxic effects.

\section{Materials and methods}

Chemicals, reagents and antibodies. Connaught strain BCG (Willowdale, Canada) was obtained from Aventis as a lyophilized preparation. Each vial was reconstituted in diluent provided by the manufacturer. Cycloheximide, 5-bromo-2deoxyuridine (Brdu) and R-phycoerythrin (PE)-conjugated rabbit anti-BCG antibody were purchased from Sigma (St. Louis, MO, USA). Fluorescein isothiocynate (FITC) was obtained from Merck (Darmstadt, Germany). Lipofectamine and Opti-Mem media were used for transfection and were purchased from Invitrogen (Carlsbad, CA, USA), also anti- 
Table I. Internalization of BCG correlates well with the amount of $\alpha 5$ receptors present.

\begin{tabular}{lccc}
\hline Cell line & \multicolumn{3}{c}{ Percentage of positive cells } \\
\cline { 2 - 4 } & $\alpha 5$ receptor & $\beta 1$ receptor & BCG internalized \\
\hline RT4 & $7.238 \pm 1.593$ & $81.77 \pm 2.11$ & $3.530 \pm 0.647$ \\
RT112 & $9.95 \pm 4.24$ & $\mathrm{ND}$ & $9.330 \pm 1.854$ \\
MGH & $40.18 \pm 1.12^{\mathrm{a}}$ & $61.51 \pm 3.31$ & $36.478 \pm 1.463^{\mathrm{a}}$ \\
\hline
\end{tabular}

aData are significantly different from RT4 and RT112; ND, not done. Data are expressed as mean \pm SEM.

Brdu was from Pharmingen (San Diego, CA, USA). Antibodies to the integrin receptors were purchased from Chemicon (Temecula, CA, USA). Antibodies to focal adhesion kinase (FAK) and phosphotyrosine were from BD Bioscience (Franklin Lakes, NJ, USA).

Cell culture. The bladder cancer cell lines, RT4, RT112 and MGH were cultured in RPMI-1640 media supplemented with $10 \%$ heat inactivated fetal bovine serum, $2 \mathrm{mM}$ l-glutamate, $50 \mathrm{U} / \mathrm{ml}$ penicillin and $50 \mu \mathrm{g} / \mathrm{ml}$ streptomycin. The cells were incubated in humidified air containing $5 \% \mathrm{CO}_{2}$ at $37^{\circ} \mathrm{C}$. Cells were seeded in 6-well plates at a density of $0.5 \times 10^{5}$ cells three days prior to the experiment. On the day of the experiment, cells were starved for $2 \mathrm{~h}$ in RPMI in the absence of serum and antibiotics. Transfection of the $\alpha 5$ containing plasmid (a kind gift from Professor F. Giancotti, Memorial Sloan-Kettering Cancer Center, New York) was performed according to the manufacturer's instructions using $2.5 \mu \mathrm{g}$ of DNA.

Preparation of labeled BCG. The reconstituted BCG was washed to ensure the removal of debris and large bacterial aggregates, which may hinder the process of bacterial internalization. The bacteria were labeled essentially as described by de Boer et al (9). In brief, $1 \mathrm{ml}$ of the reconstituted bacteria was added to $9 \mathrm{ml}$ of RPMI in a $15-\mathrm{ml}$ centrifuge tube. The resulting suspension was spun at $700 \mathrm{rpm}$ for $3 \mathrm{~min}$. The top $6 \mathrm{ml}$ was aliquoted into 6 Eppendorf tubes and spun at $12,000 \mathrm{rpm}$ for $5 \mathrm{~min}$ after which the supernatant was discarded. The bacteria were pooled and colony forming unit (CFU) were determined at $\mathrm{OD}_{440}$ before labeling with $0.3 \mathrm{mg} /$ $\mathrm{ml}$ FITC for $30 \mathrm{~min}$ at $20^{\circ} \mathrm{C}$. The labeled bacteria were washed to remove excess FITC prior to the addition on cells.

$B C G$ internalization assay. After treatment, cells were harvested and re-suspended in PBS buffer containing $0.2 \%$ albumin, $0.02 \% \mathrm{Na}$-EDTA and $0.01 \% \mathrm{NaN}_{3}$. To differentiate between internalized and surface bound $\mathrm{BCG}$, cells were incubated for $30 \mathrm{~min}$ at $4^{\circ} \mathrm{C}$ with polyclonal rabbit anti-BCG antibodies (1:100). At the end of the incubation period, the cells were washed and incubated with PE-conjugated goat anti-rabbit antibodies $(1: 100)$ for $30 \mathrm{~min}$ at $4^{\circ} \mathrm{C}$. Cells were washed and fixed in $0.5 \%$ formaldehyde and analyzed by flow cytometry. For each sample, 10,000 cells were collected.

Labeling of cells and staining with anti-Brdu. Cells $\left(1 \times 10^{6}\right)$ were stained with $10 \mu \mathrm{M}$ of Brdu in a $25 \mathrm{~cm}^{2}$ flask overnight before plating for transfection with the $\alpha 5$ containing plasmid

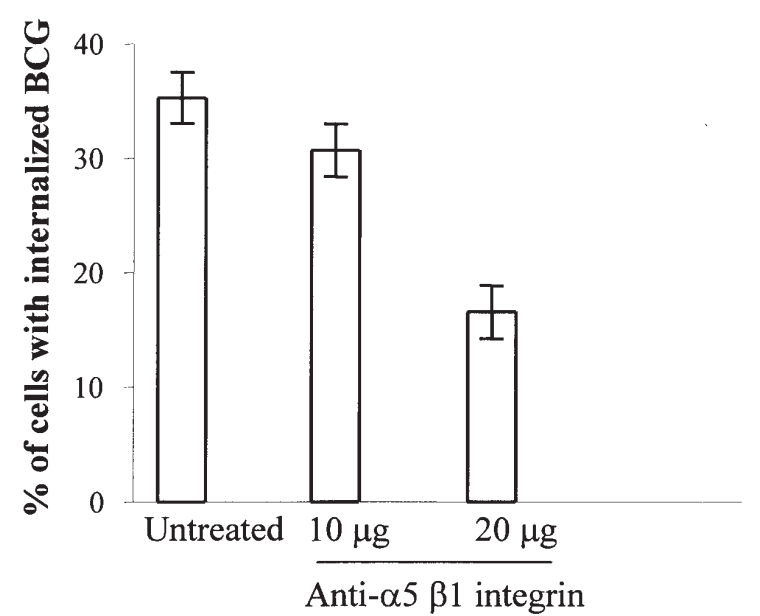

Figure 1. Blocking BCG uptake using anti-integrin antibodies. MGH cells were treated with 10 and $20 \mu \mathrm{g}$ of the anti- $\alpha 5 \beta 1$ integrin antibodies prior to treatment with BCG. Cells treated with the antibodies showed decreased internalization of BCG compared to untreated controls. Clear bars represent $\%$ of cells with internalized BCG.

DNA. Cells were transfected using lipofectamine. Twenty-four hours after transfection, cells were inoculated with $1 \times 10^{7} \mathrm{CFU}$ of bacteria. Cells were harvested the next day for staining with anti-Brdu antibody. Briefly, the pelleted cells were fixed with $70 \%$ ethanol for $20 \mathrm{~min}$. After centrifugation and washing to remove residual ethanol, the cells were re-suspended in 2 $\mathrm{N} \mathrm{HCl}$ for $20 \mathrm{~min}$. The cells were again pelleted and washed before incubation in $0.1 \mathrm{M}$ sodium borate for another $2 \mathrm{~min}$. Cells were centrifuged and re-suspended in PBS containing $0.5 \%$ Tween-20. Anti-Brdu antibody was added. After $20 \mathrm{~min}$ of incubation, cells were given a final wash prior to analysis by flow cytometry.

Receptor analysis. Cells were washed, pelleted by centrifugation and re-suspended in PBS containing 1\% BSA and $0.01 \%$ sodium azide. Antibody to the $\alpha 5$ receptors was added and incubated for $20 \mathrm{~min}$ at $4^{\circ} \mathrm{C}$. Cells were washed to remove any unbound antibody prior to the addition of a secondary antibody. After incubation for another $20 \mathrm{~min}$ at $4^{\circ} \mathrm{C}$, cells were given a final wash and fixed with $1 \%$ formaldehyde before analysis by flow cytometry.

Immunoprecipitation and immunoblotting. Cell lysate $(500 \mu \mathrm{g})$ was re-suspended in immunoprecipitation buffer containing $10 \mathrm{mM}$ Tris, pH 7.4, $150 \mathrm{mM} \mathrm{NaCl}, 1 \mathrm{mM}$ EDTA, $1 \mathrm{mM}$ 
A

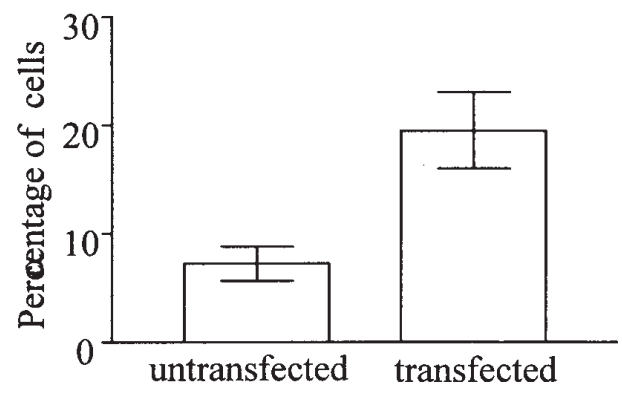

B

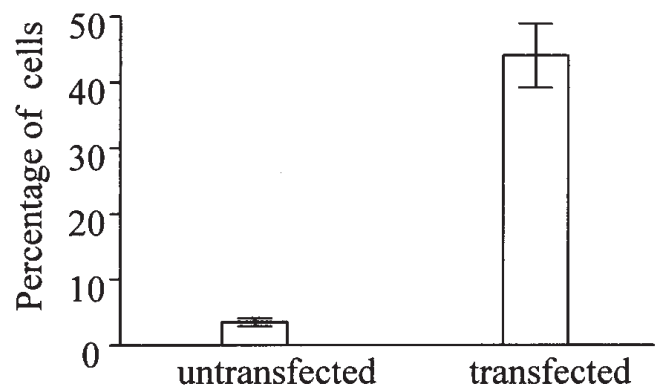

$\mathrm{C}$

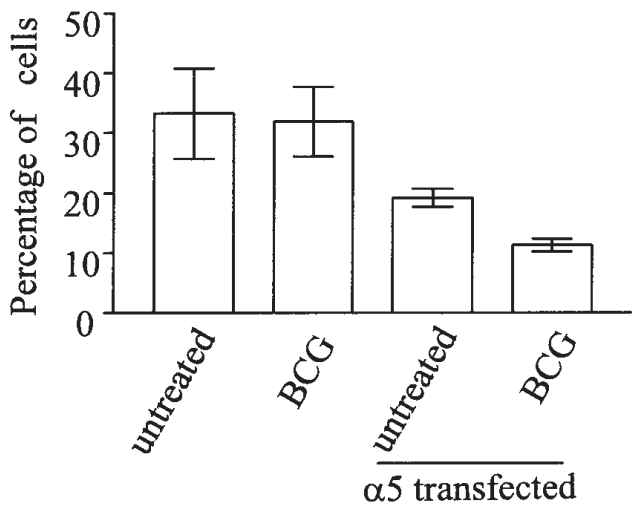

Figure 2. BCG internalization results in a decrease in cellular proliferation. RT4 cells showed increased expression of the $\alpha 5$ receptors following transfection with $\alpha 5$-containing plasmid (A). Cells transfected with the $\alpha 5$ receptor plasmid had a higher percentage of cells with internalized BCG compared to controls (B). Treatment of transfected cells with BCG rendered them susceptible to the cytotoxic effect of BCG compared to untransfected RT4 cells as shown by the percentage of anti-Brdu positive cells (C).

EGTA, $1 \%$ Triton X-100 and 0.5\% IGEPAL in the presence of protease inhibitors. After the addition of agarose beads $(20 \mu 1)$, the samples were mixed by rotation for $30 \mathrm{~min}$ at $4{ }^{\circ} \mathrm{C}$. The samples were centrifuged for $1 \mathrm{~min}$ and the supernatant was transferred to fresh tubes and incubated with anti-FAK antibodies overnight at $4^{\circ} \mathrm{C}$. Samples were incubated with $20 \mu \mathrm{l}$ of agarose beads for $1 \mathrm{~h}$ at $4^{\circ} \mathrm{C}$ the following day. After centrifugation to remove the supernatant, the beads were washed several times with immunoprecipitation buffer before boiling in Laemmli buffer. Samples were loaded onto an SDSPAGE gel. After transfer to nitrocellulose membrane and blocking with $1 \%$ BSA in Tris-buffered saline, the membrane was probed with anti-phosphotyrosine antibody that was conjugated directly to horseradish peroxidase (HRP) for 1 to $2 \mathrm{~h}$ at room temperature. After repeated washes in TBST, antibody binding was visualized using chemiluminescence.
Cycloheximide treatment and BCG internalization and cytotoxicity studies. MGH cells $\left(1 \times 10^{5}\right)$ were plated/well in 6-well plates. After $6 \mathrm{~h}$ when most of the cells were attached, the media was removed and replaced with blank media overnight. The following day, the media were replaced with complete RPMI media with $1 \mu 1$ of $10 \%$ ethanol in the control wells and $1 \mu 1$ of $1 \mathrm{mg} / \mathrm{ml}$ cycloheximide in the cycloheximide-treated wells. BCG $\left(1 \times 10^{7} \mathrm{cfu}\right)$ was added to each well and incubated with the cells at $37^{\circ} \mathrm{C}$ for 2,24 and $48 \mathrm{~h}$ after which time the cells were washed with PBS, harvested and either analyzed by flow cytometry or counted using a haemocytometer.

\section{Results}

Bladder cancer cell lines differ in their ability to ingest BCG. Three different bladder cancer cell lines were used in our study. Both RT4 and RT112 were not susceptible to the antiproliferative effect of BCG unlike MGH. The ability of these cells to internalize BCG over a $2 \mathrm{~h}$ period was monitored. This time frame was chosen because clinically patients are requested to hold $\mathrm{BCG}$ in the bladder for $2 \mathrm{~h}$ after intravesical instillation. Table I shows both RT4 and RT112 have a low level of BCG internalization compared to MGH cells. This correlated with low expression levels of the integrin $\alpha 5$ receptors on these 2 cell lines compared to MGH cells, in contrast, $B 1$ integrin receptor expression was fairly high in the cell lines (Table I). To show that the fibronectin receptors play a crucial role in BCG internalization, we treated MGH cells with anti-integrin antibodies for $2 \mathrm{~h}$ and this blocked the uptake of the bacteria by MGH cells (Fig. 1).

Transient transfection of the a5 receptor, increased internalization of BCG and cell death. Transfection of RT4 cells with a plasmid encoding the $\alpha 5$ receptor gene increased the expression of the $\alpha 5$ receptor by $\sim 2$-fold (Fig. 2A). RT4 cells, transfected with the integrin plasmid, also showed increased uptake of the bacteria over a $2 \mathrm{~h}$ inoculation period compared to its untransfected counterpart (Fig. 2B). When the viability of these transfected cells were assessed it was discovered that transfection with the $\alpha 5$ integrin did decrease cell proliferation but transfection with the $\alpha 5$ integrin gene and exposure to BCG for $24 \mathrm{~h}$ after transfection caused a further decrease in cell viability (Fig. 2C). This indicates that the susceptibility of the bladder cancer cells to the cytotoxic effects of BCG may depend on their ability to internalize the bacteria.

Internalization of BCG in contrast to its cytotoxic effects does not require the production of new mammalian proteins. To delineate the mechanism required for internalization, $\mathrm{MGH}$ cells were pre-treated with cycloheximide for $2 \mathrm{~h}$ prior to incubation with BCG for 2, 24 and $48 \mathrm{~h}$. Internalization of BCG by MGH cells was greater at 24 and $48 \mathrm{~h}$ than at $2 \mathrm{~h}$. Cycloheximide reduced but did not block BCG internalization suggesting that new protein synthesis is not required for the uptake of BCG and that receptor recycling may be sufficient for continued uptake (Table II). To ascertain if the cytotoxic effects of internalized BCG required new protein synthesis the effect of the presence of cycloheximide on cell death was 
Table II. Internalization of BCG is reduced but not blocked by cycloheximide.

\begin{tabular}{lccc}
\hline & \multicolumn{2}{c}{ Percentage of cells with internalized BCG } & $48 \mathrm{~h}$ \\
\cline { 2 - 4 } Sample & $2 \mathrm{~h}$ & $24 \mathrm{~h}$ & $0.75 \pm 0.46$ \\
Cell & $0.07 \pm 0.01$ & $0.21 \pm 0.09$ & $55.8 \pm 0.89^{\mathrm{a}}$ \\
Cell + BCG & $27.55 \pm 4.98$ & $49.3 \pm 4.69^{\mathrm{a}}$ & $1.31 \pm 0.76$ \\
Cell + Cyc & $0.07 \pm 0.03$ & $0.15 \pm 0.03$ & $45.8 \pm 2.6^{\mathrm{b}}$ \\
Cell + Cyc + BCG & $18.43 \pm 3.64$ & $32.59 \pm 3.06^{\mathrm{b}}$ & $4^{\mathrm{b}}$ \\
\hline
\end{tabular}

${ }^{a}$ Data are significantly different from cell alone; bdata are significantly different from cell + BCG; Cyc, Cycloheximide. Data are expressed as mean \pm SEM.

Table III. Cycloheximide blocks the cytotoxic effects of BCG on MGH cells.

\begin{tabular}{lccc}
\hline & \multicolumn{2}{c}{ Cell number $\left(1 \times 10^{5}\right)$} & $48 \mathrm{~h}$ \\
\cline { 2 - 4 } Sample & $2 \mathrm{~h}$ & $24 \mathrm{~h}$ & $6.75 \pm 0.16$ \\
\hline Cell & $1.24 \pm 0.123$ & $2.94 \pm 0.25$ & $3.63 \pm 0.17^{\mathrm{a}}$ \\
Cell + BCG & $1.34 \pm 0.110$ & $2.63 \pm 0.16$ & $1.59 \pm 0.1$ \\
Cell + Cyc & $1.24 \pm 0.140$ & $1.72 \pm 0.11$ & $1.67 \pm 0.16$ \\
Cell + Cyc + BCG & $1.18 \pm 0.150$ & $1.59 \pm 0.09$ & \\
\hline
\end{tabular}

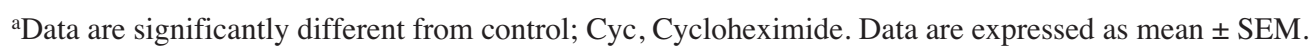

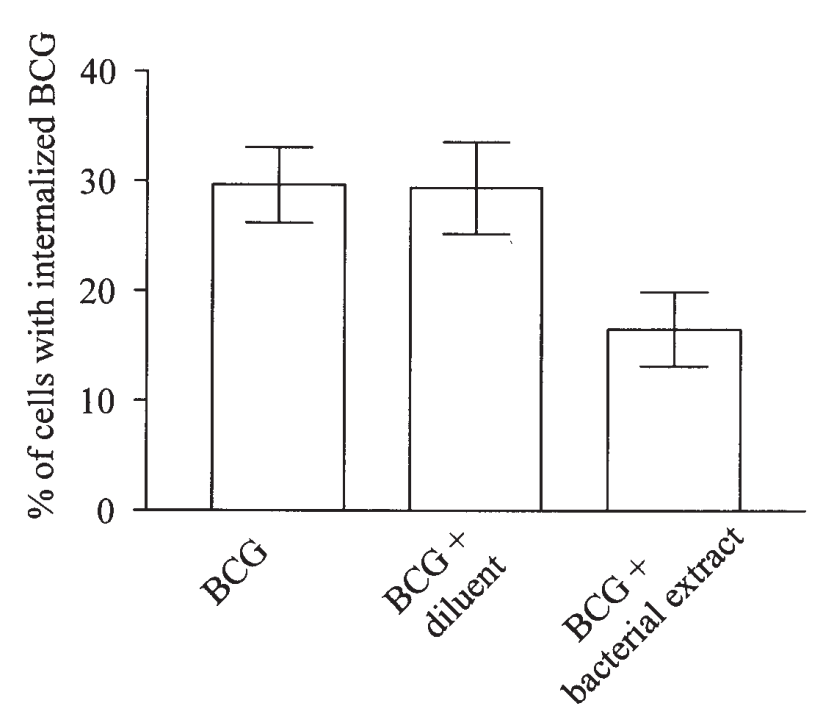

Figure 3. Pre-treatment of MGH cells with BCG extract caused a decrease in BCG internalization. MGH cells were pretreated with bacterial extract and then exposed to BCG for $2 \mathrm{~h}$. Bacterial proteins present in the extract inhibited BCG internalization.

monitored. From Table III it is clear that cycloheximide blocks not only cell proliferation but also BCG-induced cell death.

Pre-treatment of MGH cells with BCG culture extract caused a decrease in BCG internalization. Bacterial culture extract was obtained by first re-suspending the vial of lyophilized bacteria in $1 \mathrm{ml}$ of the diluent. The bacterial suspension was subsequently centrifuged to pellet the bacteria. The supernatant, containing secretary products/and bacterial extracts from the bacteria, was filtered through a $0.2-\mu$ filter to remove remaining bacteria prior to incubation with $\mathrm{MGH}$ cells. The cells were pre-treated for $2 \mathrm{~h}$ with the filtered bacterial extract before the addition of BCG for another $2 \mathrm{~h}$. Pre-treatment with the bacterial extract lowers the internalization of the bacteria compared to cells that were treated with diluent only indicating that the bacterial extract contains secreted proteins that block uptake by cells (Fig. 3).

Retardation of BCG internalization may be caused by dephosphorylation of the focal adhesion kinase (FAK). Endocytosis of bacteria by mammalian cells requires the participation and phosphorylation of a number of proteins, one of which is FAK. Since the bacterial extract was found to inhibit BCG uptake, we examined if this inhibition was mediated by affecting the phosphorylation status of FAK. MGH cells were pre-treated for $2 \mathrm{~h}$ with diluent or bacterial extract before incubation with BCG for another $2 \mathrm{~h}$. The cells were harvested, immunoprecipitated with FAK antibody and probed with anti-phosphotyrosine. Cells that were treated with bacterial extract show a lower level of tyrosine phosphorylated FAK compared to cells treated with diluent alone (Fig. 4).

\section{Discussion}

Although the use of BCG in the management of bladder cancer is reputed to be the most successful form of immunotherapy, the exact mechanism by which it exerts its anti-tumor effect is still being investigated. Studies suggest that the main cause for failing BCG therapy is the inability to mount an effective 


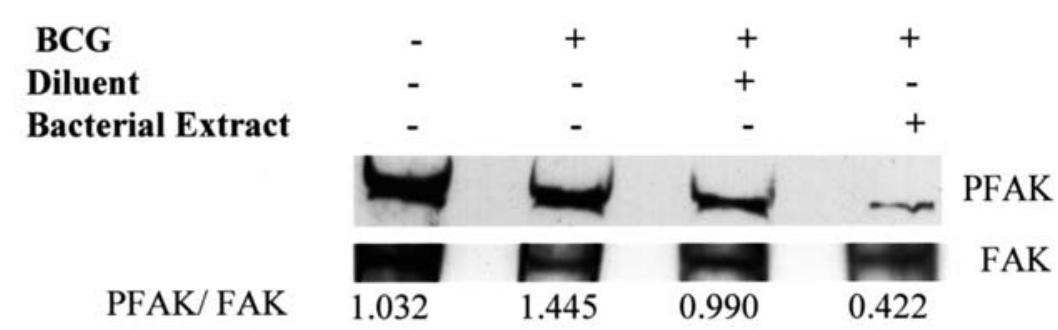

Figure 4. The bacterial extract causes dephosphorylation of FAK. A representative Western blot shows that treatment of MGH cells with the bacterial extract for $2 \mathrm{~h}$ prior to exposure to BCG resulted in dephosphorylation of FAK. Phosphorylated FAK (PFAK) and total FAK (FAK) were detected with antiphosphorylated FAK and FAK antibodies respectively. Densitometric analysis of the bands indicated that the ratio of PFAK/FAK decreased.

immunological response particularly via a failure to secrete certain cytokines (10). The immunomodulatory effect of cytokines present in the bladder of patients undergoing BCG therapy was confirmed by a number of studies both in vitro and in vivo. Kurisu et al (11) reported that incubation of bladder cancer cell lines with BCG in the presence of peripheral blood mononuclear cells inhibited the proliferation of tumor cells. The active components responsible for this inhibition are interferon- $\gamma$ and tumor necrosis factor- $\alpha$. Another in vivo study showed that there was upregulation of MHC II and ICAM I that was induced by secretion of interferon- $\gamma(12,13)$. Although great emphasis has been put on the recruitment of immune cells by BCG to the tumor site, relatively few studies have focused on the role that bladder urothelial cells play in BCG immunotherapy, although they constitute the first line of contact with the bacteria upon instillation.

In this study, we demonstrated that bladder cancer cells with different surface expression of the $\alpha 5$ receptors display a different ability to internalize BCG. MGH cells, which have the highest level of $\alpha 5$ receptors, exhibit significant internalization of the bacteria compared to 2 other cell lines, RT4 and RT112. That BCG internalization is important for its cytotoxic effect was confirmed as RT4, a cell line which was shown to be unable to internalize BCG nor respond to its anti-tumor effect (5), was rendered susceptible by transfecting it with a plasmid carrying the $\alpha 5$ gene. Bevers et al (6) also reported in a similar study that bladder cancer cells have different capacity for internalizing BCG although they related this to the grade of the tumor rather than the level of receptor expression. Binding of BCG to the fibronectin receptor can have effects on cellular signaling via activation of AP-1 and NF- $\kappa \mathrm{B}$ transcription factors $(14,15)$ while internalized BCG can bring about phenotypical changes to the urothelial cells such as upregulation in the levels of MHC class II molecules, ICAM I and B7-1 enabling it to function like an antigenpresenting cell (7).

The first study to link the initiation of an immune response with fibronectin-mediated attachment of BCG was performed by Ratliff et al (3). Since then, further studies carried out on animals have reaffirmed that binding of BCG to the bladder urothelia is necessary for the expression of BCG-induced anti-tumor effect. Increased attachment of BCG to the bladder urothelia by the use of a fibrin clot stabilizer was shown to significantly influence anti-tumor efficacy (16). The mycobacterial protein, FAP, which is responsible for the attachment to fibronectin was also characterized (17). The internalization of BCG which causes necrosis of tumor cells results in the release of cellular content into the tumor milieu thereby providing proteins for antigen presentation by the antigen presenting cells (18). Hence, both direct and bystander effects can play a role in BCG immunotherapy.

Since internalization can influence a cell's response to BCG, we went on to further characterize events that may influence cellular BCG uptake. Treatment with cycloheximide, an inhibitor of protein synthesis, indicated that there is no need for the synthesis of new mammalian proteins in the internalization process. Rather uptake occurs via receptor recycling. However, BCG-induced killing was blocked by the presence of cycloheximide. This effect indicates that new protein synthesis is required for the induction of necrosis or can be due to the known ability of cycloheximide to reduce cellular reactive oxygen species (ROS) which we had previously shown to correlate with the cytotoxic effect of BCG on cells (8).

Pathogens are known to secrete phosphatases to inhibit engulfment by immune cells. A well-studied example is that of Yersinia pseudotuberculosis which prevents its internalization by injection of cellular phosphatases into mammalian cells. Injection of the phosphatases appears to be a rapid event with dephosphorylation of mammalian proteins observed as early as 5 min (19). Mycobacteria are known to secrete a number of proteins into the extracellular media (20). That BCG, like Yersinia, secretes phosphatases is supported by studies whereby two genes with sequence homology to protein tyrosine phosphatases were cloned from the genome of Mycobacteria tuberculosis and both sequences were also found to be present in Mycobacteria bovis (21). When the bladder cancer cells were incubated with the filtered bacterial extract derived from the reconstitution of lyophilized BCG prior to the incubation with the bacteria, internalization was prevented. Inhibition of internalization correlated well with the loss of phosphorylation of FAK which is a major mediator of the integrin pathway and also involved in the uptake of bacteria such as Yersinia (19).

Our data indicate that if the direct cytotoxic effects of BCG on bladder cancer cells are desired during therapy, it is necessary for reconstituted freeze-dried BCG to be repeatedly washed to remove enzymes in the preparation which can interfere with the internalization of BCG. The advantage of BCG internalization is that the necrosis of cancer cells ensures the release of material that can be taken up by dendritic cells and so cause immune stimulation. BCG is known to induce a fairly non-specific immune response but antigen presentation 
by dendritic cells can engender the production of specific immune responses. In conclusion, BCG exerts a direct cytotoxic effect on bladder cancer cells and this response depends on whether the cells have the ability to internalize the bacteria.

\section{Acknowledgements}

This study was funded by grants from the National Medical Research Council of Singapore (NMRC/0493/2000 and NMRC/0828/2004).

\section{References}

1. Netto Junior NR, Levi d'Ancona CA, Claro JF and Ilari O: Immunoprophylaxis of superficial bladder cancer: a prospective and randomized comparison of oral versus intravesical Bacillus Calmette-Guerin. Arch Esp Urol 44: 1025-1028, 1991.

2. Ratliff TL, Kavoussi LR and Catalona WJ: Role of fibronectin in intravesical BCG therapy for superficial bladder cancer. J Urol 139: 410-414, 1998.

3. Ratliff TL, Palmer JO, McGarr JA and Brown EJ: Intravesical Bacillus Calmette-Guerin therapy for murine bladder tumor: initiation of the response by fibronectin-mediated attachment of Bacillus Calmette-Guerin. Cancer Res 47: 1762-1766, 1987.

4. Zhao W, Schorey JS, Bong-Mastek M, Ritchey J, Brown EJ and Ratliff TL: Role of a bacillus Calmette-Guerin fibronectin attachment protein in BCG-induced antitumor activity. Int J Cancer 86: 83-88, 2000.

5. Zhang Y, Khoo HE and Esuvaranathan $\mathrm{K}$ : Effects of Bacillus Calmette-Guerin and interferon-alpha-2B on human bladder cancer in vitro. Int J Cancer 71: 851-857, 1997.

6. Bevers RFM, de Boer EC, Kurth K-H and Schamhart DHJ: BCG-induced Interleukin-6 upregulation and BCG internalization in well and poorly differentiated human bladder cancer cell lines. Eur Cytokine Netw 9: 181-186, 1998.

7. Ikeda N, Toida I, Iwasaki A, Kawai K and Akaza H: Surface antigen expression on bladder tumor cells induced by bacillus Calmette-Guerin (BCG): a role of BCG internalization into tumor cells. Int J Urol 9: 29-35, 2002.

8. Pook SH, Esuvaranathan K and Mahendran R: N-acetylcysteine augments the cellular redox changes and cytotoxic activity of internalized mycobacterium bovis in human bladder cancer cells. J Urol 68: 780-785, 2002.

9. de Boer EC, Bevers RF, Kurth KH and Schamhart DH: Double fluorescent flow cytometric assessment of bacterial internalization and binding by epithelial cells. Cytometry 25: 381-387, 1996.
10. Luo Y, Chen X and O'Donnell MA: Role of Th1 and Th2 cytokines in BCG-induced IFN-gamma production: cytokine promotion and simulation of BCG effect. Cytokine 21: 17-26, 2003.

11. Kurisu H, Matsuyama H, Shimabukuro T and Naito K: Cytokine-mediated antitumor effect of bacillus Calmette-Guerin on tumor cells in vitro. Cancer Immunol Immunother 34: 249253, 1994.

12. Jackson AM, Alexandroff AB, McIntyre M, Esuvaranathan K, James K and Chisholm GD: Induction of ICAM 1 expression on bladder tumors by BCG immunotherapy. J Clin Pathol 47: 309-312, 1994.

13. Prescott S, James K, Busuttil A, Hargreave TB, Chisholm GD and Smyth JF: HLA-DR expression by high-grade superficial bladder cancer treated with BCG. Br J Urol 63: 264-269, 1989.

14. Chen F, Zhang G, Iwamoto Y and See WA: Bacillus CalmetteGuerin initiates intracellular signaling in a transitional carcinoma cell line by cross-linking $\alpha 5 \beta 1$ integrin. J Urol 170: 605-610, 2003.

15. Chen FH, Crist SA and Zhang GJ: Interleukin-6 production by human bladder tumor cell lines is up-regulated by BCG through NF- $\kappa \mathrm{B}$ and AP-1 via an immediate early pathway. J Urol 168: 786-797, 2002.

16. Hudson MA, Brown EJ, Ritchey JK and Ratliff TL: Modulation of fibronectin-mediated Bacillus Calmette-Guerin attachment to murine bladder mucosa by drugs influencing the coagulation pathways. Cancer Res 51: 3726-3732, 1991.

17. Schorey JS, Holsti MA, Ratliff TL, Allen PM and Brown EJ: Characterization of the fibronectin-attachment protein of Mycobacterium avium reveals a fibronectin-binding motif conserved among mycobacteria. Mol Microbiol 21: 321-329, 1996.

18. Barker RN, Erwig LP, Hill KS, Devine A, Pearce WP and Rees AJ: Antigen presentation by macrophages is enhanced by the uptake of necrotic, but not apoptotic cells. Clin Exp Immunol 127: 220-225, 2002.

19. Persson C, Carballeira N, Wolf-Watz H and Fallman M: The PTPase YopH inhibits uptake of Yersinia, tyrosine phosphorylation of p130Cas and FAK, the associated accumulation of theses proteins in peripheral focus adhesions. EMBO J 16: 2307-2318, 1997.

20. Raynaud C, Etiene G, Peyron P, Laneele MA and Daffé M: Extracellular enzyme activities potentially involved in the pathogenicity of Mycobacterium tuberculosis. Microbiology 144: 577-587, 1998.

21. Koul A, Choidas A, Treder M, Tyagi AK, Drlica K, Singh Y and Ullrich A: Cloning and characterization of secretory tyrosine phosphatases of Mycobacterium tuberculosis. J Bacteriol 182: 5425-5432, 2000. 